\title{
Ab interno tube ligation for refractory hypotony following non-valved glaucoma drainage device implantation
}

\author{
A. Vergados ${ }^{1,2} \cdot$ A. A. Mohite ${ }^{1} \cdot$ Velota C. T. Sung $^{1}$ (I) \\ Received: 26 February 2019 / Revised: 4 May 2019 / Accepted: 17 June 2019 / Published online: 22 July 2019 \\ (C) The Author(s) 2019
}

\begin{abstract}
Purpose To report the 2-year outcomes of a novel surgical technique allowing reduction of the intraluminal diameter of the tube without total tube occlusion in order to allow enough increase in outflow resistance to permit resolution of hypotony whilst also achieving adequate IOP control.

Methods This was a single-surgeon retrospective case note review of all non-valved GDD cases over an 8-year period (20082015) that underwent ab interno ligation of the drainage tube in order to manage post-operative hypotony (Baerveldt or Molteno). Twelve eyes of 12 patients (4.4\%) developing refractory hypotony that did not respond to multiple intracameral ophthalmic viscoelastic device (OVD) injections were included in this retrospective case series and were treated with our ab interno tube ligation technique. The post-ligation management algorithm consisted of re-instating topical anti-glaucoma agents, laser suture lysis (LSL), or further ab interno ligation.

Results Mean IOP increased from $2.8 \mathrm{mmHg}$ at baseline to $7.8 \mathrm{mmHg}, 7.1 \mathrm{mmHg}, 9.0 \mathrm{mmHg}, 13.6 \mathrm{mmHg}, 10.9 \mathrm{mmHg}$, $13.9 \mathrm{mmHg}$ and $13.6 \mathrm{mmHg}$ at day 1 , week 1 , month 1 , month 3, month 6 , year 1 and year 2 respectively, with or without additional topical anti-glaucoma medications. Although hypotony resolution following our technique was achieved in all eyes at 2 years, $8.3 \%$ of cases required reinstatement of topical medications to maintain IOP control within the target range.

Conclusions We propose ab interno partial tube tying as an effective surgical option to achieve an immediate, predictable and sustained IOP elevation either as a primary procedure or when traditional methods have failed to resolve hypotony in eyes with non-valved GDDs.
\end{abstract}

Keywords Non-valved glaucoma drainage device $\cdot$ Hypotony $\cdot$ Glaucoma $\cdot$ Tube ligation

\section{Introduction}

Ocular hypotony following non-valved glaucoma drainage device (GDD) implantation can lead to sight-threatening hypotony-related complications (HRCs). The management can be challenging, requiring injections of ocular viscoelastic devices (OVDs) [1], total occlusion of the drainage tube using a

A. Vergados and A. A. Mohite contributed equally to this work.

A. Vergados and A. A. Mohite are joint first authors.

Velota C. T. Sung

velota.sung@nhs.net

1 Birmingham and Midland Eye Centre, City Hospital, Sandwell and West Birmingham Hospitals NHS Trust, Dudley Road, Birmingham B18 7QH, UK

2 Whipps Cross University Hospital, Whipps Cross Road, London E11 1NR, UK ligating suture, or removal of the drainage tube from the anterior chamber [2].

Total ligation or removal of the tube may however cause excessive increase in intraocular pressure (IOP) and further jeopardize the already compromised optic nerve in these eyes. There is often a volatility in the fluctuation of IOP in these complex eyes, which are challenging to manage without causing serious permanent damage to vision. Restoring the fine balance between aqueous production and outflow is hence paramount in the successful treatment of refractory hypotony following non-valved GDD implantation.

We therefore developed a novel ab interno partial ligation technique that aims to reduce the intraluminal diameter of the tube without total tube occlusion, so that outflow resistance is increased enough to allow resolution of hypotony without causing a rebound spike in IOP. To the best of the authors' knowledge, this is the first study to describe such a technique and report its long-term outcomes in eyes with refractory 
hypotony following either Baerveldt or Molteno GDD implantation for complex glaucoma.

\section{Methods}

\section{Patient selection}

This was a single-surgeon retrospective case note review of all non-valved GDD cases over an 8-year period (2008-2015) that underwent ab interno ligation of the drainage tube in order to manage post-operative hypotony. All cases were refractory to multiple fixed-volume intracameral injections of sodium hyaluronate $1.4 \%$ at the slit-lamp, our preferred initial intervention for early post-operative hypotony after non-valved GDDs [1]. Either a Baerveldt (Abbott Laboratories Inc., Abbott Park, IL, USA) or a Molteno (Molteno Ophthalmic Ltd., Dunedin, New Zealand) drainage device was used in all cases. Baerveldt implants were either $250 \mathrm{~mm}^{2}$ or $350 \mathrm{~mm}^{2}$ in size depending on the ocular history and risk of post-operative hypotony.

A standard surgical technique was performed for implantation of the GDD in all cases, as has been previously described [3]. In brief, all cases had placement of a full-length intraluminal occluding 3/0 polyamide stent suture (Supramid, S. Jackson Inc., Alexandria, VA, USA) as well as external tube ligation with a $6 / 0$ polyglactin absorbable suture, to prevent excessive early postoperative filtration. All cases also had a 0.5-mm-long Sherwood tube fenestration slit made just anterior to the occlusive $6 / 0$ ligature to allow some modulation of early post-operative IOP until dissolution of the ligature in about week 6 .

\section{Ab interno tube ligation technique}

Two 9/0 prolene sutures were tied around the intracameral portion of the tube using slip-knots. For the first tie a $6 / 0$ prolene suture was placed within the tube lumen whilst tightening the knot, and then removed. The second tie was placed in the same way, but using a $7 / 0$ prolene suture within the tube lumen, as illustrated in the schematic (Fig. 1). This was done under the operating microscope. Each intraluminal prolene suture acted as a 'spacer', allowing a consistent graduated reduction of the tube gauge, with the proximal portion of the tube becoming narrower than its distal portion. The aim was to achieve a graduated reduction in the intraluminal diameter of the tube without total tube occlusion.

The ties were placed intra-camerally without externalizing the tube, via two paracenteses on either side of the tube entry site (Fig. 2a). Another paracentesis was made at the limbus opposite to the tube position to allow the intraluminal prolene suture to pass straight into the lumen of the tube (Fig. 2a). A $9 / 0$ prolene suture needle was passed through from one paracentesis to exit the second paracentesis by docking into a 25-gauge needle introduced via the second paracentesis. The 25 -gauge needle then guided the prolene suture needle out of the second paracentesis, leaving the suture passing on the posterior side of the tube. Using an intraocular manipulator (Sung manipulator, Altomed Limited, Tyne and Wear, UK), the $9 / 0$ prolene suture was then looped over the tube and brought out through the first paracentesis similar to the Siepser slip-knot technique [4]. A three-throw slip-knot was then fashioned outside the eye, slid inside the anterior chamber and tied tightly with 2 micro needle-holders on the posterior side of the tube. The tightness of the knot was tested by pulling the intra-luminal spacer suture gently to make sure that the knot was tight enough to narrow the lumen to the size of the spacer suture. Another double-throw knot was then tied to lock the knots in position. The threads of the suture were then cut with 23 -gauge intra-ocular scissors. The first $6 / 0$ spacer suture was then removed from the tube lumen and replaced with a $7 / 0$ prolene spacer suture. The whole procedure was then repeated with another $9 / 0$ prolene suture tied more tightly and nearer to the proximal end of the tube (Fig. 2b). The 7/0 prolene spacer suture was then removed from the tube lumen. In some cases, a third total tie was placed at the most proximal end without an intra-luminal spacer suture to achieve total occlusion of the tube, depending on the extent of hypotony. Trypan blue dye was injected through the proximal tube opening using a 30-gauge cannula to check for total tube occlusion in cases where a third total occlusion tie was placed, and was confirmed when no dye was observed to pass beyond this tie (Fig. 2c).

\section{Post-ligation management}

We followed a step-wise management strategy following this procedure. If IOP was deemed too high, pressure-lowering medications were then introduced back into the treatment regime. If the IOP was still too high, the proximal tighter tie (nearer to the end of the tube in AC) was cut using neodymium-doped yttrium aluminium garnet laser suture lysis (LSL) in the outpatient clinic or surgically in theatre. If, however, the IOP did not increase following the procedure, a further totally occluding $9 / 0$ prolene was tied around the tube without the use of an intra-luminal spacer suture as described earlier. This final tie could then also be released at a later date using LSL should the IOP then rebound too high.

Success was defined as resolution of hypotony with or without a rebound rise in IOP above $22 \mathrm{mmHg}$, and resolution of hypotony related complications. Complications of the procedure were recorded at each time point, including uncontrolled IOP rise requiring LSL, untying of one or more ligatures, or repeat ab interno tube ligation for unresolved hypotony. Persistent hypotony, choroidal or retinal detachments, endophthalmitis or phthisis, as well as development of 


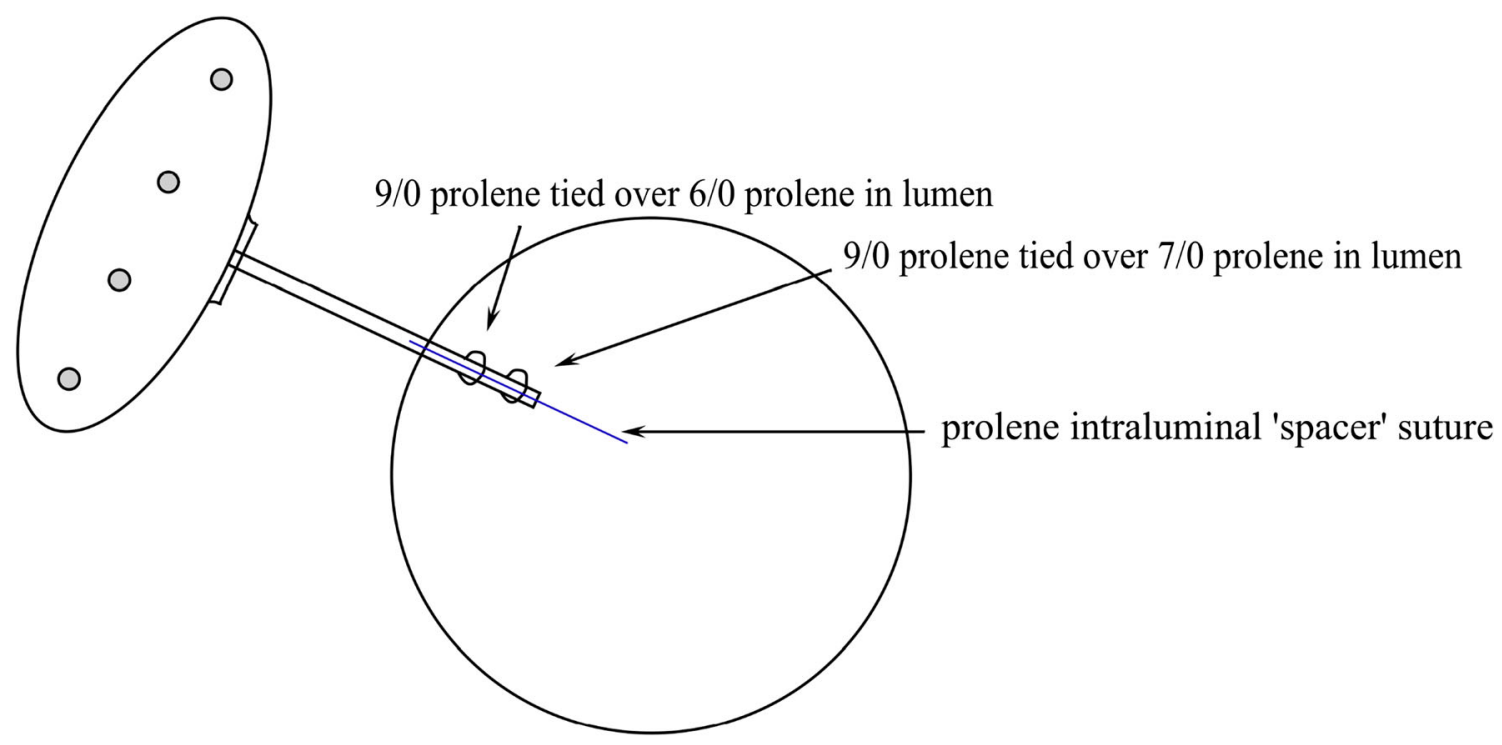

Fig. 1 Schematic of partial internal tube tying technique

visually significant cataracts, cystoid macular oedema (CMO) or epiretinal membrane (ERM) were recorded over a 2-year follow-up period.

The study was part of a larger retrospective study of the outcomes of glaucoma surgical intervention approved by the local Research Ethics Committee (reference: 12/WM/0158) and adhered to the tenets of the Declaration of Helsinki. Descriptive statistical analyses were performed using IBM SPSS Statistics version 23.0.

\section{Results}

During the study period, 271 non-valved (Baerveldt or Molteno implants) GDD implant surgeries were performed by the authors' team (VS). Of these, 12 eyes of 12 patients (4.4\%) developed refractory hypotony that did not respond to multiple intracameral OVD injections and were included in this retrospective case series. Four cases were in paediatric patients ( $<18$ years of age at the time of GDD surgery). Table 1 shows baseline patient demographics.
All eyes were on maximum tolerated medical therapy for their glaucoma prior to undergoing GDD implantation, with a mean of $4.3( \pm 0.9)$ anti-glaucoma medications and a mean cup: disc ratio of $0.8( \pm 0.1)$. Prior to tube-tying, 10 eyes (83.3\%) had choroidal detachment or maculopathy due to refractory hypotony.

Mean IOP increased from $2.8 \mathrm{mmHg}( \pm 2.3)$ prior to $\mathrm{ab}$ interno tube ligation to $7.8 \mathrm{mmHg}( \pm 5.8)$ at day 1 . This rise persisted long-term with resolution of hypotony in all cases (Fig. 3), with mean IOP measuring $7.1 \mathrm{mmHg}( \pm 6.4)$, $9.0 \mathrm{mmHg}( \pm 7.8), 13.6 \mathrm{mmHg}( \pm 5.8), 10.9 \mathrm{mmHg}( \pm 9.4)$, $13.9 \mathrm{mmHg}( \pm 6.7)$ and $13.6 \mathrm{mmHg}( \pm 6.5)$ at week 1 , month 1 , month 3 , month 6 , year 1 and year 2 respectively, with or without additional topical anti-glaucoma medications. Hypotony resolution at 2 years was achieved in all eyes at 2 years, with no significant rebound IOP rise in $83.3 \%$ (10 eyes). Topical medications to maintain IOP control within target were reinstated in $16.7 \%$ ( 2 eyes). The change in BCVA over time is shown in Fig. 4.

Table 2 shows the number of additional interventions required over 2 years of follow-up following this technique.



Fig. 2 Intra-operative images showing a the $6 / 0$ prolene spacer suture, $\mathbf{b}$ a second tie with $9 / 0$ prolene suture and $\mathbf{c}$ confirmation of total occlusion which was required in some cases, via injection of trypan blue dye. White arrows $=$ tube ties. Black arrow $=$ intraluminal spacer suture 
Table 1 Patient demographics and clinical characteristics

\section{General}

\begin{tabular}{|c|c|}
\hline Eyes, $n$ & 12 \\
\hline Males/females, $n(\%)$ & $7(58) / 5(42)$ \\
\hline Mean age, years, (range) & $33.9(8.6-66.0)$ \\
\hline Mean time to partial tube ligation, months, $( \pm \mathrm{SD})$ & $3.3( \pm 3.7)$ \\
\hline \multicolumn{2}{|l|}{ Primary glaucoma diagnosis, $n,(\%)$} \\
\hline Uveitic & $5(42)$ \\
\hline JIA & 2 \\
\hline Steroid induced & 1 \\
\hline FHC & 1 \\
\hline Sympathetic panuveitis & 1 \\
\hline Neovascular (due to PDR) & $2(17)$ \\
\hline Angle recession & $2(17)$ \\
\hline Post-vitrectomy and oil & $1(8)$ \\
\hline Pseudoexfoliation & $1(8)$ \\
\hline Aphakic glaucoma/nanophthalmos & $1(8)$ \\
\hline \multicolumn{2}{|l|}{ Previous incisional surgery, $n(\%)$} \\
\hline Trabeculectomy surgery & $3(25)$ \\
\hline ECCE & $1(8)$ \\
\hline Phacoemulsification & $5(42)$ \\
\hline Pars plana vitrectomy & $1(8)$ \\
\hline Previous TSCD treatment, $n(\%)$ & $4(33)$ \\
\hline Of which 1 prior TSCD & 2 \\
\hline Of which 2 prior TSCD & 1 \\
\hline Of which 3 prior TSCD & 1 \\
\hline In neovascular glaucoma & 2 \\
\hline In angle recession & 1 \\
\hline In pseudoexfoliation & 1 \\
\hline In uveitic eyes & 0 \\
\hline \multicolumn{2}{|l|}{ Type of GDD, $n(\%)$} \\
\hline Molteno & $2(17)$ \\
\hline Baerveldt $350 \mathrm{~mm}^{2}$ & $5(42)$ \\
\hline Baerveldt $250 \mathrm{~mm}^{2}$ & $5(42)$ \\
\hline MMC not used, $n(\%)$ & $2(17)$ \\
\hline MMC used, $n(\%)$ & $10(83)$ \\
\hline $0.2 \mathrm{mg} / \mathrm{ml}$ for $3 \mathrm{~min}$ & 6 \\
\hline $0.4 \mathrm{mg} / \mathrm{ml}$ for $3 \mathrm{~min}$ & 3 \\
\hline $0.4 \mathrm{mg} / \mathrm{ml}$ for $5 \mathrm{~min}$ & 1 \\
\hline
\end{tabular}

$G D D$, glaucoma drainage device; $P D R$, proliferative diabetic retinopathy; $J I A$, juvenile idiopathic arthritis; $F H C$, Fuch's heterochromic cyclitis; $T S C D$, trans-scleral ciliary body cyclodiode

Only one case $(8.3 \%)$ resolved without any further procedures. Five $(41.6 \%)$ required further OVD injections into the AC. Two-thirds (66.7\%) required further partial or total tying of tube with the same ab interno technique. Two-thirds $(66.7 \%)$ required untying of one or more sutures either surgically or by LSL to lower the IOP. The hypotony resolved in all cases before the end of the 24-month follow-up period. One eye developed retinal detachment likely secondary to previous trauma, and there were no cases of phthisis or endophthalmitis. Of the five phakic eyes, four $(80 \%)$ required cataract surgery after tube tying. Of these, two eyes were rendered aphakic whilst the other two had intraocular lens implantation. Visual acuity improved in all four eyes undergoing cataract extraction, with a mean pre- and post-phacoemulsification BCVA of $1.34 \pm 0.6$ and $0.49 \pm 0.5 \log$ MAR respectively.

\section{Discussion}

We achieved IOP control without the need to recommence any anti-glaucoma medications in $83.3 \%$ (10/12) of eyes. Two eyes required recommencement of medications. The first had a history of juvenile glaucoma and was commenced back on glaucoma drops at year 2 (fixed combination of dorzolamide/ timolol $0.5 \%$ and bimatoprost $0.01 \%$ ), though this was still significantly fewer than the four topical agents plus oral acetazolamide that was required originally. This patient required total tube ligation due to persistent cystoid macular oedema. The second had a history of microphthalmos and vitrectomy with silicone oil tamponade in situ following retinal detachment, and required a fixed combination of brinzolamide/ timolol to achieve target IOP having been on four topical agents plus oral acetazolamide prior to GDD implantation.

Visual acuities did not significantly improve despite resolution of hypotony in our series. In some cases, visual acuity actually worsened. This was due to the development of visually significant cataracts in $80 \%$ of the phakic eyes in our series (4 of 5 eyes) as well as retinal detachment requiring vitrectomy and oil in one eye. One pseudophakic eye required laser capsulotomy for posterior capsular opacification, two eyes developed significant ERM (of which one later underwent pars plana vitrectomy), and one eye developed early post-operative CMO treated successfully with orbital floor triamcinolone. This same eye developed corneal decompensation and later underwent endothelial lamellar keratoplasty 5 years after tube tying. There were no other cases of corneal decompensation in our series, and hypotony related maculopathy or choroidal detachments resolved in all cases. It is important to also note that all eyes had advanced glaucomatous optic neuropathy which would contribute to the gradual decline in BCVA over the postoperative follow-up period.

The Ahmed versus Baerveldt Study demonstrated that nonvalved glaucoma tube shunts achieve a greater reduction in IOP and glaucoma medications at 5 years, as well as lower failure rates when compared to valved shunts [5]. However, non-valved shunts have a greater 5-year risk of hypotony and its related complications when compared to valved shunts [5, 6]. Although early hypotony following non-valved GDDs has been reported to occur in as much as $39 \%$ of eyes $[3,7]$, the incidence of persistent hypotony is far less common, with reports in the literature ranging from $0 \%$ to $13 \%$, which is in 
Fig. 3 Mean IOP and number of anti-glaucoma medications before tube surgery, prior to partial tying of tube, and at various time points after partial tying of tube. Error bars represent $95 \%$ confidence intervals

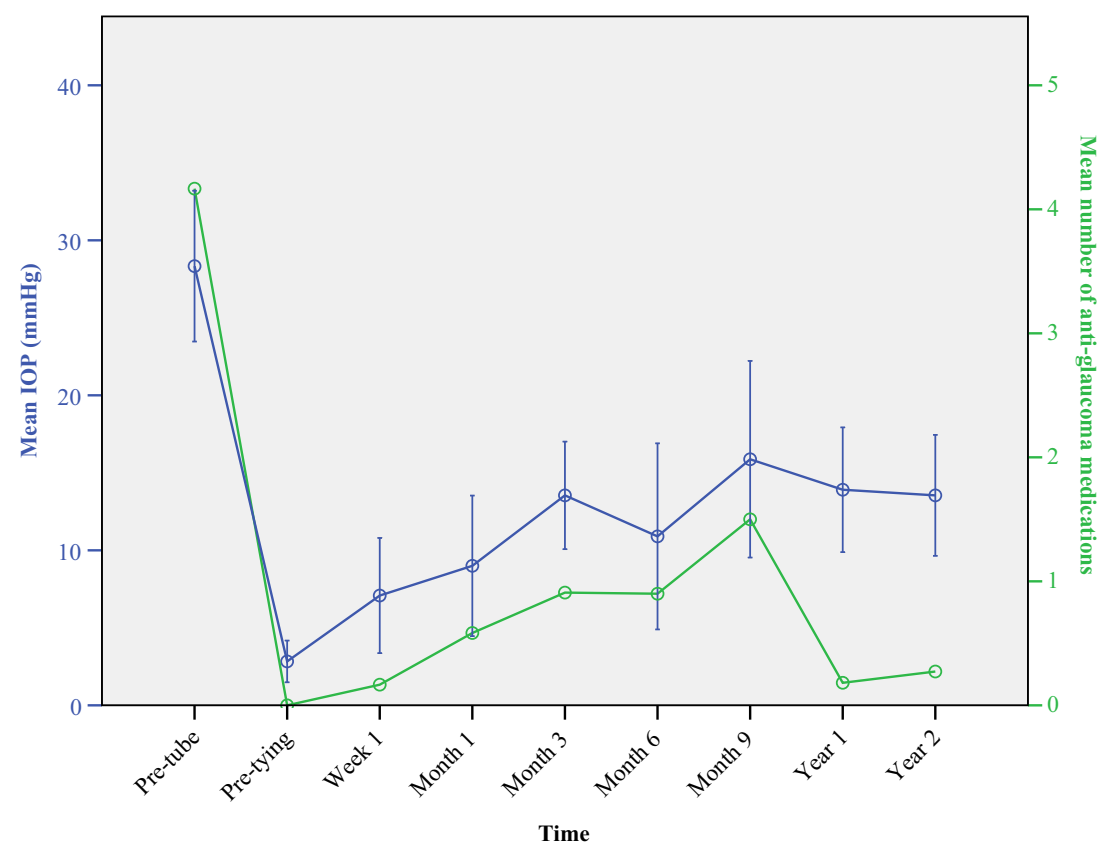

keeping with our rate of $4.4 \%[6,8-10]$. The variation is likely to be due to a difference in case-mix between studies, with those studies having higher proportions of complex eyes (chronic uveitis, trauma, prior cyclo-destructive procedures or neovascular glaucoma) being more likely to have greater rates of persistent hypotony.

Despite the well-known risk of persistent hypotony after GDD surgery, few studies have reported on the outcomes of surgical management of these cases. Common practice is to use intracameral injections of OVD or total ligation of the external portion of the drainage tube using non-absorbable sutures [8]. The former option may require multiple injections of OVD due to their short-lived effect, although more viscous devices such as reticulated hyaluronic acid have recently been used to prevent phthisis and maintain aesthetic appearance in pre-phthisical blind eyes with chronic hypotony [11]. One third of our cases had previous cyclo-destructive procedures, which may explain the poor resolution of hypotony in these eyes using fixed-volumes of repeated intracameral OVD injections.

Recent studies have described a controlled reduction of outflow restriction using LSL after Baerveldt tube surgery and graduated intraluminal stent removal to achieve low rates of hypotony-related complications [8]. Techniques used to
Fig. 4 Mean best-corrected $\log$ MAR visual acuity (BCVA) before tube surgery, prior to partial tying of tube, and at various time points after partial tying of tube. Error bars represent 95\% confidence intervals

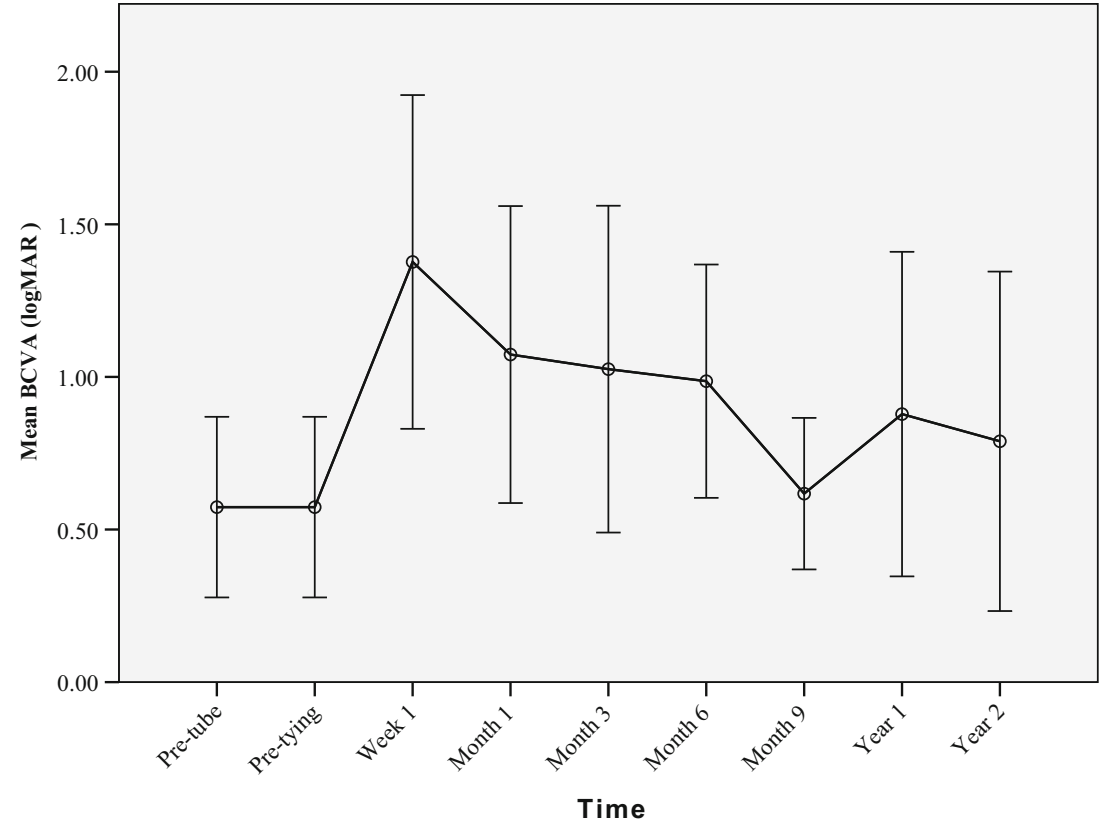


Table 2 Additional surgical interventions required per case after ab interno partial tying of tube

\begin{tabular}{|c|c|c|c|c|c|}
\hline $\begin{array}{l}\text { Patient } \\
\text { age (years) }\end{array}$ & Diagnosis & GDD implanted & $\begin{array}{l}\text { Number of } \\
\text { prior TSCD }\end{array}$ & Additional post-ligation interventions & $\begin{array}{l}\text { Number of } \\
\text { additional } \\
\text { interventions }\end{array}$ \\
\hline 8.6 & Uveitic (JIA related) & Molteno & 0 & $\begin{array}{l}\text { Tube repositioning/Repeat partial tube ligation } \\
\text { (month 21) }\end{array}$ & 1 \\
\hline 11.8 & Uveitic (JIA related) & BVT 250 & 0 & $\begin{array}{l}\text { Untying of proximal tube tie (month 3) } \\
\text { Repeat partial tube ligation (month 4) } \\
\text { Repeat partial tube ligation (month 5) } \\
\text { LSL of proximal tube tie (month 5) }\end{array}$ & 4 \\
\hline 13.1 & $\begin{array}{l}\text { Uveitic (sympathetic } \\
\text { panuveitis) }\end{array}$ & BVT 250 & 0 & $\begin{array}{l}\text { Repeat partial tube ligation (month 3) } \\
\text { Lensectomy/PPV/untying of distal tie (month 7) }\end{array}$ & 2 \\
\hline 36.5 & Uveitic (FHC) & Molteno & 0 & $\begin{array}{l}\text { Additional partial ligation with a 3rd proximal } \\
\text { tie (week } 3 \text { ) } \\
\text { LSL of } 2 \text { proximal tube ties (week } 3 \text { ) } \\
\text { AC paracentesis } \times 8 \text { (week } 3 \text { ) } \\
\text { Repeat partial tube ligation of } 2 \text { distal tube ties } \\
\text { (week 6) }\end{array}$ & 4 \\
\hline 59.1 & Uveitic (steroid induced) & BVT 350 & 0 & $\begin{array}{l}\text { HGV into AC (week 1) } \\
\text { Repeat partial tube ligation (week 2) } \\
\text { AC paracentesis (week 2) } \\
\text { LSL of proximal tube tie (week 4) } \\
\text { HGV into AC (week 5) }\end{array}$ & 5 \\
\hline 14.5 & Angle recession & BVT 250 & 1 & $\begin{array}{l}\text { HGV into AC (week 5) } \\
\text { PPV/oil for exudative RD (month 7) } \\
\text { ROSO as oil blocking tube (month 8) } \\
\text { Untying of proximal tube tie (month 12) }\end{array}$ & 4 \\
\hline 66.0 & Angle recession & BVT 350 & 0 & $\begin{array}{l}\text { HGV into AC (month 5) } \\
\text { Repeat partial tube ligation with } 3 \times 9 / 0 \\
\quad \text { (month } 16 \text { ) } \\
\text { Re-insertion of supramid } \\
\text { Repeat partial tube ligation (year 2) }\end{array}$ & 3 \\
\hline 18.1 & Post-vitrectomy + oil & BVT 250 & 0 & $\begin{array}{l}\text { Untying of distal tube tie (month 7) } \\
\text { LSL of proximal tube tie (month 10) }\end{array}$ & 2 \\
\hline 29.7 & NVG & BVT 350 & 2 & Nil & 0 \\
\hline 33.3 & NVG & BVT 250 & 3 & $\begin{array}{l}\text { LSL of proximal tube tie (month 11) } \\
\text { Repeat partial tube ligation (month 11) } \\
\text { Lensectomy (month 9) }\end{array}$ & 3 \\
\hline 53.7 & Aphakic glaucoma & BVT? 350 & 0 & $\begin{array}{l}\text { HGV into AC (week 1, week 2, week 5) } \\
\text { Phaco/IOL/GSL (month 4) }\end{array}$ & 4 \\
\hline 62.1 & $\mathrm{PXF} / \mathrm{JOAG}$ & BVT 350 & 1 & $\begin{array}{l}\text { HGV into AC (week 4, week 5, week } 8 \text {, week } \\
9 \text {, week 12) } \\
\text { Total internal tube ligation (month 3) } \\
\text { LSL of tube tie (month 5) } \\
\text { Phaco/IOL/anterior vitrectomy (month 22) }\end{array}$ & 8 \\
\hline
\end{tabular}

$J I A$, juvenile idiopathic arthritis; $F H C$, Fuch's heterochromic uveitis; $N V G$, neovascular glaucoma; $P X F$, pseudoexfoliation glaucoma; JOAG, juvenile open angle glaucoma; $G D D$, glaucoma drainage device; $B V T$, Baerveldt tube; $T S C D$, trans-scleral cyclodiode; $L S L$, laser suture lysis; $A C$, anterior chamber; $H G V$, Healon GV; $P P V$, pars plana vitrectomy; ROSO, removal of silicone oil; Phaco, phacoemulsification

treat eyes with HRC in this series included intracameral injections of OVD and re-insertion of the intraluminal Supramid stent. A recent case report describes the ab interno placement of two 5/0 nylon sutures to stent an Ahmed glaucoma valve without the need for intracameral tube tying in an eye with neovascular glaucoma [12], with good 6-month outcomes. Another paper describes the use of a 3/0 Supramid suture inserted ab interno for two cases of persistent hypotony, but with limited long-term data [13], whilst another study describes ab externo insertion of the Xen gel stent into the
GDD tube lumen of two cases with chronic hypotony [14]. However, these techniques alone may not always resolve hypotony, particularly in very high-risk cases with volatile aqueous production rates [15].

In spite of our graduated technique of narrowing the tube diameter, it was still difficult to predict which eyes would recover immediately from the hypotony. We found that $40 \%$ still required further OVD injections and two-thirds required further $\mathrm{ab}$ interno tube tying, mostly with the addition of a total occlusion tie, to achieve early hypotony resolution. 
This would suggest that a total occlusion tie is necessary in the majority of cases to completely stop aqueous drainage, allowing ciliary body function and thus the IOP to recover. Conversely when the hypotony did recover, two-thirds of eyes required subsequent LSL or untying of one or more suture ties over the following months because of raised IOP. We believe this is to be expected because of the nature of such complex eyes, in which there is a fine, individualized, and volatile balance between the degree of ciliary body dysfunction, aqueous production, and outflow resistance.

Having a clear step-wise management strategy with close patient follow-up allowed us to respond to changes in IOP in a methodical manner whilst avoiding serious complications. Our LSL technique allows selective and stepwise release of the ties to minimize the risk of recurrent hypotony in these eyes. The delicate balance in these eyes is further reflected by the fact that despite our stepwise strategy, three cases $(25 \%)$ required repeat ab interno tying of the tube.

Although some surgical techniques to deal with refractory hypotony have been described in academic meetings, to the best of our knowledge there have been no publications on their medium to long-term outcomes. Those described either require conjunctival dissection prior to ligation of the external portion of the tube, or externalization of the intracameral portion of the tube via a full-thickness corneal incision over the tube opening. The former technique carries the risk of inciting further conjunctival inflammation and scarring, and makes later adjustment of this ligating suture difficult if the IOP then elevates excessively. The latter technique can potentially alter the tube position causing tube adhesion to the cornea and subsequent corneal endothelial damage, given that studies have shown accelerated rates of endothelial cell loss when the tube is positioned close to the endothelium [16]. In addition, a short corneal wound to gain access to the tube makes water-tight wound closure difficult, increasing the risk of leakage and its associated complications including epithelial ingrowth.

We used Mitomycin C (MMC) during GDD implantation in $83 \%$ of eyes in this series, based on a previous study that demonstrated that intraoperative use of MMC was not significantly associated with hypotony or low IOP at 3 and 5 years [3]. Our experience is that early hypotony within the first 3 months is due to over-drainage rather than MMC use.

The main weakness of this study is its retrospective nature and the relatively small number of the cases. However, it would take a long time to accumulate a substantially larger sample size given the low reported rates of persistent hypotony and our considerable experience in developing a safe GDD implantation technique aimed at keeping hypotony rates to a minimum.

\section{Conclusions}

We developed a novel-graduated ab interno tube ligation technique and post-ligation management strategy to manage refractory hypotony in high-risk eyes fitted with non-valved GDDs without the need for conjunctival incisions or externalization of the tube. Our series demonstrates high safety and efficacy at medium-term follow-up and we therefore propose our technique as an effective option to achieve immediate and sustained IOP elevation either as a primary procedure or when traditional methods (intracameral viscoelastic injections or reinsertion of intraluminal stents) have failed to resolve hypotony in eyes with non-valved GDDs.

\section{Compliance with ethical standards}

Conflict of interest The authors declare that they have no conflict of interest.

Ethical approval For this type of retrospective study, formal consent was not required. All procedures performed were in accordance with the ethical standards of the local research committee and with the 1964 Helsinki declaration and its later amendments.

Open Access This article is distributed under the terms of the Creative Commons Attribution 4.0 International License (http:// creativecommons.org/licenses/by/4.0/), which permits unrestricted use, distribution, and reproduction in any medium, provided you give appropriate credit to the original author(s) and the source, provide a link to the Creative Commons license, and indicate if changes were made.

\section{References}

1. Chiam PJ, Chen X, Haque MS, Sung VC (2018) Outcome of fixed volume intracameral sodium hyaluronate $1.4 \%$ injection for early postoperative hypotony after Baerveldt glaucoma implant. Clin Exp Ophthalmol 46:1035-1040

2. Stein JD, McCoy AN, Asrani S, Herndon LW, Lee PP, McKinnon SJ, Allingham RR, Challa P (2009) Surgical management of hypotony owing to overfiltration in eyes receiving glaucoma drainage devices. J Glaucoma 18:638-641

3. Mandalos A, Sung V (2017) Glaucoma drainage device surgery in children and adults: a comparative study of outcomes and complications. Graefes Arch Clin Exp Ophthalmol 255:1003-1011

4. Siepser SB (1994) The closed chamber slipping suture technique for iris repair. Ann Ophthalmol 26:71-72

5. Christakis PG, Kalenak JW, Tsai JC, Zurakowski D, Kammer JA, Harasymowycz PJ, Mura JJ, Cantor LB, Ahmed II (2016) The Ahmed versus Baerveldt study: five-year treatment outcomes. Ophthalmology 123:2093-2102

6. Budenz DL, Barton K, Gedde SJ, Feuer WJ, Schiffman J, Costa VP, Godfrey DG, Buys YM, the Ahmed Baerveldt Comparison Study Group (2015) Five-year treatment outcomes in the Ahmed Baerveldt comparison study. Ophthalmology 122:308-316

7. Siegner SW, Netland PA, Urban RC Jr, Williams AS, Richards DW, Latina MA, Brandt JD (1995) Clinical experience with Baerveldt glaucoma drainage implant. Ophthalmology 102:1298-1307 
8. Sharkawi E, Artes PH, Oleszczuk JD, Bela C, Achache F, Barton K, Bergin C (2016) Systematic occlusion of shunts: control of early postoperative IOP and hypotony-related complications following glaucoma shunt surgery. J Glaucoma 25:54-61

9. Nguyen QH, Budenz DL, Parrish RK 2nd (1998) Complication of Baerveldt glaucoma drainage implants. Arch Ophthalmol 116:571-575

10. Ceballos EM, Parrish RK II, Schiffman JC (2002) Outcome of Baerveldt glaucoma drainage implants for the treatment of uveitic glaucoma. Ophthalmology 109:2256-2260

11. Stead RE, Juma Z, Turner S, Jones LD, Sung VC (2016) A novel use of reticulated hyaluronic acid (Healaflow) for hypotony eyes in patients with uveitis. Br J Ophthalmol 100:727-730

12. Lim SH, Hwang YH (2018) Management of postoperative ocular hypotony after tube surgery by stenting using two 5-0 nylon threads. Korean J Ophthalmol 32:523-524

13. Kuruvilla S, Landers J, Craig JE (2016) Secondary stenting of glaucoma drainage implant: a novel technique for treatment of late hypotony. Clin Exp Ophthalmol 44:860-861
14. Valimaki J (2018) Xen gel stent to resolve late hypotony after glaucoma drainage implant surgery: a novel technique. J Glaucoma 27: 926-928

15. Aujla JS, Lee GA, Vincent SJ, Thomas R (2013) Incidence of hypotony and sympathetic ophthalmia following trans-scleral cyclophotocoagulation for glaucoma and a report of risk factors. Clin Exp Ophthalmol 41:761-772

16. Tan AN, Webers CA, Berendschot TT, de Brabander J, de Witte PM, Nuijts RM, Schouten JS, Beckers HJ (2017) Corneal endothelial cell loss after Baerveldt glaucoma drainage device implantation in the anterior chamber. Acta Ophthalmol 95:91-96

Publisher's note Springer Nature remains neutral with regard to jurisdictional claims in published maps and institutional affiliations. 